The Open Automation and Control
Bentham open
CrossMark
Content list available at: www.benthamopen.com/TOAUTOCJ/
DOI: $10.2174 / 1874444301608010006$

\title{
Smooth Switching Tracking Control for a Class of Linear Time- Varying Systems with Input Quantization and Input Saturation
}

\author{
Zhubing $\mathrm{Hu}^{*}$, Gaohua Cai and Lifu Du \\ ${ }^{I}$ Department of Electrical and Electronic Engineering, Chengde Petroleum College Chengde, 067000, P. R. China \\ ${ }^{2}$ Beijing Aerospace Automatic Control Institute, Beijing 100854, P. R. China
}

\begin{abstract}
This paper deals with the problem of robust smooth switching state feedback controller design for a class of linear timevarying systems with input quantization and input saturation, which is a common case in the practical engineering for modern flight vehicle. First, the problem statement is presented, in which a tracking problem is converted into a regulation problem by constructing a deviation system, the tracking error deviation is treated as the system performance and the conditions for smooth switching tracking control are discussed based on the theory of robust control technique. Secondly, the state feedback gain of the deviation system with input saturation and input quantization is designed by solving Linear Matrix Inequalities (LMIs) based convex optimization problem with the conditions for smooth switching tracking control being considered. Finally, validation simulations are carried out for the longitudinal dynamics model of hypersonic vehicle driven by Reaction Control System (RCS) to illustrate the effectiveness and rationality of the proposed approach.
\end{abstract}

Keywords: Input Quantization and Input Saturation, Linear Matrix Inequalities, Multi-Model, Switched System, Time-varying System.

\section{INTRODUCTION}

In optimizing autopilot design and evaluating robustness for flight vehicle, many significant advances have been achieved with newly developed control theory during the recent years [1 - 6]. While, developing an effective approach is still a challenging issue, especially for an aircraft with multiple flight conditions and each of the condition corresponding to a different model, especially as the hypersonic vehicle driven by Reaction Control System (RCS) during initial reentry phase. Reaction Control Systems (RCS) consisting of thrusters that can fire to produce rolling, pitching and yawing moments have been used for reentry vehicle control for decades. The RCS control authority is relatively constant across a dynamic pressure envelope, and the valve dynamics of the RCS thrusters may be significantly faster than the motor dynamics of aerosurfaces. The RCS of hypersonic vehicle can be characterized as pulsed control effectors with two states (on and off) and can be described as an actuator with quantization and saturation characteristics [7]. In this way, hypersonic reentry vehicle driven by RCS becomes a plant with input quantization and input saturation. However, it is a challenge to design a command tracking control system for such a plant theoretically. And also, an efficient control law is required to meet a desired performance, when the hypersonic reentry vehicle driven by RCS transits from one flight condition to another.

Phase plane method [8 - 11] and pulse width modulation (PWM) method $[12,13]$ are the traditional control techniques for vehicle driven by RCS. Phase plane method is an effective tool for designing single-axis control system for reentry vehicles, such as the Apollo command module [8] and the Mars Science Laboratory [11]; while, it cannot be applied to systems with rotational coupling. When the duty cycles of the thrusters of RCS are modulated by PWM, the thrusters can be treated as continuous actuators. By such an approach, the continuous multivariable control methods

\footnotetext{
* Address correspondence to this author at the Department of Electrical and Electronic Engineering, Chengde Petroleum College Chengde, 067000, P. R. China; Tel: 0314-2375666; E-mail: hubing9973316@163.com
} 
could be used to design the control system for flight vehicle. While, PWM method may lead to rapid cycling of the thrusters, which can result in excessive wear on the RCS propellant control valves. In our recent work [14], an approach based on Linear Matrix Inequalities (LMIs) convex optimization procedure is proposed for designing the CommandTracking-Control-System for plant with hybrid actuator, in which, the controllers are designed at trim point for each plant, not for multi-plants. Ref. [15] presents a method to design a smooth switching gain-scheduled linear parameter varying (LPV) controller for LPV systems, such that it has a smooth transition of controllers. Ref. [16] proposed a scheme considered embeds a finite family of pre-designed controllers and a high-level unit which selects, at each instant of time, the candidate controller to be placed in feedback to the uncertain plant. While, for a class of time-varying systems with input quantization and saturation, both Refs. $[15,16]$ are not mentioned and need for further research.

Our work is related to the work of Refs. $[17,18]$, where such a novel smooth switching approach based on Linear Matrix Inequalities (LMIs) is proposed for a class of time-varying systems with input quantization and saturation, which has not been handled in the literatures to date. Based on the Lyapunov stability theory and robust control technique, a state feedback gain of the time-varying systems with input saturation and input quantization is designed by solving LMIs based convex optimization problem with the conditions for smooth switching tracking control being considered. With the controller, the closed-loop system can track a given reference signal with desired performance during the whole operating range. Most importantly, in the switching process when the flight condition changes, the system chattering can be considerably reduced by this designed constant controller. As far as we know, the smooth switching tracking control problem for time-varying systems with input saturation and input quantization has not been considered in literatures.

The paper is organized as follows. In section 2, the problem statement is presented, in which a tracking problem is converted to a regulation problem by constructing a deviation system, the tracking error deviation is treated as the system performance and the conditions for smooth switching tracking control are presented. In Section 3, the procedure for designing a state feedback gain for the deviation system with input saturation and input quantization is provided with the consideration of smooth switching conditions. And then, validation simulations are carried out for the longitudinal dynamics model of hypersonic vehicle driven by RCS in Section 4. Finally, the paper is concluded in Section 5.

\section{PROBLEM STATEMENT}

Considering the time-varying system as follows:

$$
\left\{\begin{array}{l}
\dot{x}_{p}(t)=A_{p}(t) x_{p}+B_{p}(t) u \\
z_{p}(t)=H_{p}(t) x_{p}
\end{array}\right.
$$

with system matrix $A_{p}(\mathrm{t})$, control matrix $B_{p}(\mathrm{t})$ and system performance output matrix $H_{p}(\mathrm{t})$, which include timevarying parameters. And the changing patterns of the time-varying parameters in $A_{p}(\mathrm{t}), B_{p}(\mathrm{t})$ and $H_{p}(\mathrm{t})$ are so complicated that it is difficult to obtain the analytical forms of them. Generally, in the field of engineering practice, a common method is to select some characteristic time points in the flight envelop and design controllers for each subsystems. However, such design method cannot guarantee the stability of the original time-varying model due to the negligence of the majority unconsidered operating points. The validation of global stability using this method involves infinite operating points, it is unrealistic.

To overcome the difficulties caused by the traditional method for the global stable controller design problem, a novel approach is introduced in this paper. For this purpose, we rewrite system (1) as the following form:

$$
\left\{\begin{array}{l}
\dot{x}_{p}(t)=A_{p}(\theta) x_{p}+B_{p}(\theta) u \\
z_{p}(t)=H_{p}(\theta) x_{p}
\end{array}\right.
$$

It is assumed that a finite number of discrete characteristic time points during the whole operating region of the system can be selected. At each characteristic time point, a time invariant linear system can be obtained, and the system matrix, control matrix and performance output matrix for each linear system are $A_{p i}, A_{p i}$ and $H_{p i}$, respectively, where $i=$ $1,2, \ldots N$. And also, the system matrix of system (2) can be respected by a convex combination of $A_{p i}, A_{p i}$ and $H_{p i}$, that is 


$$
A_{p}(\theta)=\sum_{i=1}^{N} \theta_{i} A_{p i}, \quad B_{p}(\theta)=\sum_{i=1}^{N} \theta_{i} B_{p i}, \quad H_{p}(\theta)=\sum_{i=1}^{N} \theta_{i} H_{p i}
$$

where $\theta=\left[\begin{array}{llll}\theta_{1} & \theta_{2} & \ldots & \theta_{N}\end{array}\right]^{T} \in \mathbf{R}^{N}$, satisfying

$$
\theta \in \Theta \triangleq\left\{\theta \in \mathbf{R}^{N}: \theta_{i}>0, \sum_{i=1}^{N} \theta_{i}=1\right\}
$$

Considering the schematic diagram of tracking control system as Fig. (1). In Fig. (1), the dynamics model of compensator is

$$
\dot{w}(t)=F_{w} w(t)+G_{w} e(t)
$$

where $\mathrm{w}(\mathrm{t})$ is the state of compensator, $e(\mathrm{t})=r(\mathrm{t})-Z_{p}(\mathrm{t})$ is the tracking error.

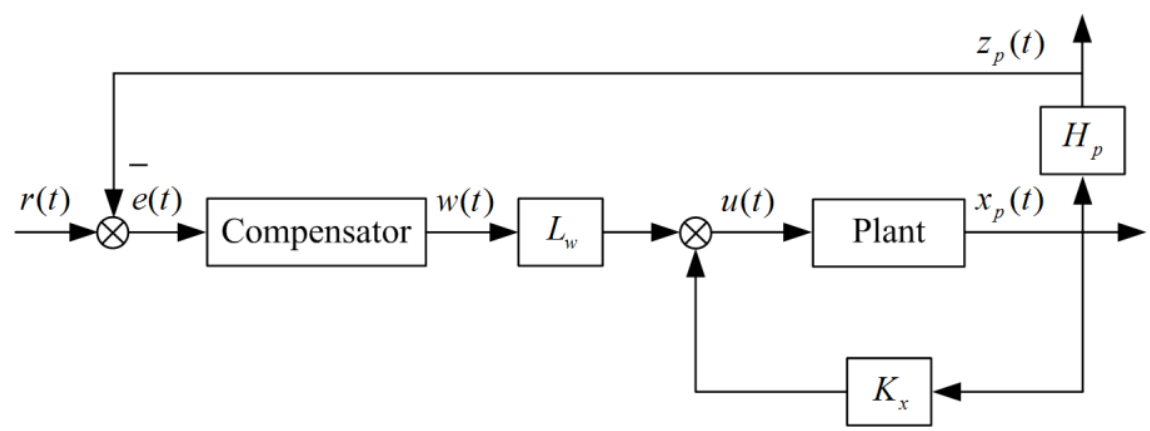

Fig. (1). Schematic diagram of tracking control system.

According to $e(\mathrm{t})=r(\mathrm{t})-Z_{p}(\mathrm{t})$, the plant model and the compensator model can be written in an augmented form as:

$$
\left\{\begin{array}{l}
{\left[\begin{array}{c}
\dot{x}_{p}(t) \\
\dot{w}(t)
\end{array}\right]=\left[\begin{array}{cc}
A_{p}(\theta) & 0 \\
-G_{w} H_{p}(\theta) & F_{w}
\end{array}\right]\left[\begin{array}{c}
x_{p}(t) \\
w(t)
\end{array}\right]+\left[\begin{array}{c}
B_{p}(\theta) \\
0
\end{array}\right] u(t)+\left[\begin{array}{c}
0 \\
G_{w}
\end{array}\right] r(t)} \\
z(t)=\left[\begin{array}{ll}
H_{p}(\theta) & 0
\end{array}\right]\left[\begin{array}{c}
x_{p}(t) \\
w(t)
\end{array}\right]
\end{array}\right.
$$

The control input can be expressed as:

$$
u(t)=\left[\begin{array}{ll}
K_{x} & L_{w}
\end{array}\right]\left[\begin{array}{c}
x_{p}(t) \\
w(t)
\end{array}\right]=K_{x} x_{p}(t)+L_{w} w(t)
$$

By redefining the state, the output, and the matrix variables to streamline the notation, the augmented equations that contain the dynamics of both the plant and the compensator are of the form

$$
\left\{\begin{array}{l}
\dot{x}(t)=A(\theta) x(t)+B(\theta) u(t)+G r(t) \\
z(t)=H(\theta) x(t)
\end{array}\right.
$$

The control input $u(\mathrm{t})=K x(\mathrm{t})$, where,

$$
A(\theta)=\left[\begin{array}{cc}
A_{p}(\theta) & 0 \\
-G_{w} H_{p}(\theta) & F_{w}
\end{array}\right], B(\theta)=\left[\begin{array}{c}
B_{p}(\theta) \\
0
\end{array}\right], G=\left[\begin{array}{c}
0 \\
G_{w}
\end{array}\right], H(\theta)=\left[\begin{array}{cc}
H_{p}(\theta) & 0
\end{array}\right], K=\left[\begin{array}{ll}
K_{x} & L_{w}
\end{array}\right]
$$


Let,

$$
A_{i}=\left[\begin{array}{cc}
A_{p i} & 0 \\
-G_{w} H_{p i} & F_{w}
\end{array}\right], B_{i}=\left[\begin{array}{c}
B_{p i} \\
0
\end{array}\right], H_{i}=\left[\begin{array}{ll}
H_{p i} & 0
\end{array}\right]
$$

Obviously, the following expressions are satisfied.

$$
A(\theta)=\sum_{i=1}^{N} \theta_{i} A_{i}, \quad B(\theta)=\sum_{i=1}^{N} \theta_{i} B_{i}, \quad H(\theta)=\sum_{i=1}^{N} \theta_{i} H_{i}
$$

Using the equations $u(\mathrm{t})=K x(\mathrm{t})$ and $\dot{x}(t)=A(\theta) x(t)+B(\theta) u(t)+G r(t) \square$ the closed-loop system is found to be:

$$
\dot{x}(t)=[A(\theta)+B(\theta) K] x(t)+G r(t)=A_{c}(\theta) x(t)+G r(t)
$$

Denote $\bar{x}$ and $\bar{u}$ be the steady-state values of the system state and control input, then the state and control deviations are given by

$$
\tilde{x}(t)=x(t)-\bar{x}, \quad \tilde{u}(t)=u(t)-\bar{u}=K(t) x-K \bar{x}=K \tilde{x}(t)
$$

Since in any acceptable design, the closed-loop plant will be asymptotically stable and $A_{c}$ is nonsingular, then according to Eq.(8), we have $A_{c}(\theta) \bar{x}+G r_{0}=0$ at steady state, so that the steady-state response $\bar{x}$ is

$$
\bar{x}=-\left[A_{c}(\theta)\right]^{-1} G r_{0}
$$

Assume that the reference command $r(\mathrm{t})$ is a step command with magnitude $\mathrm{r}_{0}$, using $x(t)=\tilde{x}(t)+\bar{x}=\tilde{x}(t)-\left[A_{c}(\theta)\right]^{-1} G r_{0}$, the closed-loop dynamics of the state deviation are seen to be

$$
\dot{\tilde{x}}(t)=A_{c}(\theta) \tilde{x}(t)
$$

Denote $\bar{e}$ as the steady-state value of the tracking error, then one can obtain that

$$
\tilde{e}(t)=e(t)-\bar{e}=[\delta-H(\theta) x(t)]-[\delta-H(\theta) \bar{x}(t)]=-H(\theta) \tilde{x}(t)
$$

According to $A_{c}(\theta)=A(\theta)+B(\theta) K$ and $\tilde{u}(t)=K \tilde{x}$, the command tracking problem has been converted to a regulator problem for the deviation system

$$
\dot{\tilde{x}}(t)=A(\theta) \tilde{x}(t)+B(\theta) \tilde{u}(t)
$$

with tracking error deviation as:

$$
\tilde{e}(t)=-H(\theta) \tilde{x}(t)
$$

Consider the regulation system involving input quantization and input saturation, Eq. (12) can be described by:

$$
\dot{\tilde{x}}(t)=A(\theta) \tilde{x}(t)+B(\theta) \operatorname{sat}[q u a(\tilde{u}(t))]
$$

where for any $\tilde{u} \in \mathbf{R}^{m}$, the quantizer function qua (.) is a piece wise constant mapping, which is defined as

$$
\text { qua }(\tilde{u})=\left\{\begin{array}{cc}
0, & -\Delta \leq \tilde{u} \leq \Delta \\
k \Delta, & k \Delta \leq \tilde{u} \leq(k+1) \Delta, \quad i=1,2, \ldots, m \\
-k \Delta, & -(k+1) \Delta \leq \tilde{u} \leq-k \Delta
\end{array}\right.
$$

where $\Delta$ is positive for all $i=1,2, \ldots m$ and represents the quantization error bound. The static uniform quantizer qua 


$$
-\Delta \leq q u a(\tilde{u})-\tilde{u} \leq \Delta, i=1,2, \ldots, m
$$

Given any vector $\tilde{u} \in \mathbf{R}^{m}$, the saturation map sat (.) is classically defined from the symmetric saturation function having level as the positive vector $\mathrm{u}_{\mathrm{s}} \mathrm{q}$

$$
\operatorname{sat}(\tilde{u})=\operatorname{sign}(\tilde{u}) \min \left\{u_{s},|\tilde{u}|\right\}, i=1,2, \ldots, m
$$

Define $\psi(K \tilde{x}(t))=q u a(K \tilde{x}(t))-K \tilde{x}(t)$ and $\phi(K \tilde{x}(t))=\operatorname{sat}(q u a(K \tilde{x}(t)))-q u a(K \tilde{x}(t))$, Eq. (14) can be expressed as follows $\square$

$$
\dot{\tilde{x}}(t)=[A(\theta)+B(\theta) K] \tilde{x}(t)+B(\theta)[\phi(K \tilde{x}(t))+\psi(K \tilde{x}(t))]
$$

where $\phi(K \tilde{x}(t))+\psi(K \tilde{x}(t))=\operatorname{sat}(q u a(K \tilde{x}(t)))-K \tilde{x}(t)$ is the error caused by quantization and saturation.

If $\phi(K \tilde{x}(t))+\psi(K \tilde{x}(t))$ is treated as the disturbance input of the system, and $\tilde{e}(t)=-H(\theta) \tilde{x}(t)$ is treated as the system performance output, the closed-loop system can be rewritten as $\square$

$$
\left\{\begin{array}{l}
\dot{\tilde{x}}(t)=[A(\theta)+B(\theta) K] \tilde{x}(t)+B(\theta)[\phi(K \tilde{x}(t))+\psi(K \tilde{x}(t))] \\
\tilde{e}(t)=-H(\theta) \tilde{x}(t)
\end{array}\right.
$$

The closed-loop system (18) can be described as the following diagram shown in Fig. (2).

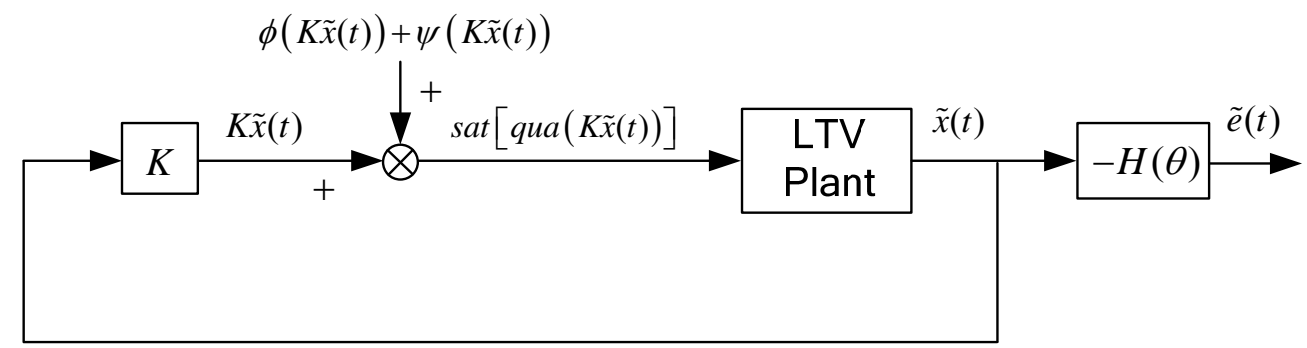

Fig. (2). Diagram of the closed-loop system.

Assume that there is no disturbance input $\phi(K \tilde{x}(t))+\psi(K \tilde{x}(t))$, system (18) is a continuous system. In order to improve the system performance with disturbance input $\phi(K \tilde{x}(t))+\psi(K \tilde{x}(t))$, the $H_{\infty}$ norm of the transfer function from $\phi(K \tilde{x}(t))+\psi(K \tilde{x}(t))$ to $e(\tilde{t})$ should be less than $\gamma_{l}$, that is $\square$

$$
\left\|G_{\theta}(s)\right\|_{\infty}<\gamma_{1}
$$

where

$$
G_{\theta}(s)=\left(\begin{array}{c:c}
A(\theta)+B(\theta) K & B(\theta) \\
\hdashline-H(\theta) & 0
\end{array}\right)
$$

And furthermore, so as to eliminate the effect of disturbance input $\phi(K \tilde{x}(t))+\psi(K \tilde{x}(t))$ on $\tilde{e}(t)$ when the model parameter changes, the following expression should be satisfied

$$
\frac{\left\|\tilde{e}\left(\beta_{m}\right)-\tilde{e}\left(\beta_{n}\right)\right\|_{\infty}}{\|\phi(K \tilde{x}(t))+\psi(K \tilde{x}(t))\|_{\infty}}<\gamma_{2}
$$

where $\beta_{m}=\left[\begin{array}{llll}\theta_{m 1} & \theta_{m 2} & \ldots & \theta_{m N}\end{array}\right]^{T}, \quad \beta_{n}=\left[\begin{array}{llll}\theta_{n 1} & \theta_{n 2} & \ldots & \theta_{n N}\end{array}\right]^{T}, \quad \beta_{m}, \beta_{n} \in \Theta, m \neq n$ 
Let $\tilde{e}\left(\beta_{m}\right)=G_{\beta_{m}}(s)[\phi(K \tilde{x}(t))+\psi(K \tilde{x}(t))]$ and $\tilde{e}\left(\beta_{n}\right)=G_{\beta_{n}}(s)[\phi(K \tilde{x}(t))+\psi(K \tilde{x}(t))]$, then

$$
\tilde{e}\left(\beta_{m}\right)-\tilde{e}\left(\beta_{n}\right)=\left[G_{\beta_{m}}(s)-G_{\beta n}(s)\right] \cdot[\phi(K \tilde{x}(t))+\psi(K \tilde{x}(t))]
$$

One can get that $\square$

$$
\left\|G_{\beta_{m}}(s)-G_{\beta n}(s)\right\|_{\infty}<\gamma_{2}
$$

where

$$
G_{\beta_{m}}(s)-G_{\beta n}(s)=\left(\begin{array}{cc:c}
A\left(\beta_{m}\right)+B\left(\beta_{m}\right) K & 0 & B\left(\beta_{m}\right) \\
0 & A\left(\beta_{n}\right)+B\left(\beta_{n}\right) K & B\left(\beta_{n}\right) \\
\hdashline-H\left(\beta_{m}\right) & H\left(\beta_{n}\right) & 0
\end{array}\right)
$$

The problem we intend to solve can be summarized as: determining a state feedback gain $K$ to stabilize the closedloop system (14) and realize smooth switching control, such that the conditions (19) and (20) should be satisfied.

\section{SMOOTH SWITCHING CONTROL DESIGN WITH INPUT QUANTIZATION AND SATURATION}

With the saturation function and quantization function defined in the above section, the following two Assumptions can be obtained by using the modified sector condition [17].

Assumption 1: Consider a matrix $R \in \mathbf{R}^{m \times n}$, the nonlinearity $\phi(\tilde{u})=\operatorname{sat}(\tilde{u})-\tilde{u}$ satisfies:

$$
\phi(\tilde{u})^{T} T_{1}(\operatorname{sat}(\tilde{u})+R \tilde{x}) \leq 0
$$

for any diagonal positive matrix $T_{1} \in \mathbf{R}^{m \times m}$, where $\tilde{x} \in S\left(u_{s}\right)$ is defined by $\square$

$$
S\left(u_{s}\right)=\left\{\tilde{x} \in \mathbf{R}^{n} ;-u_{\mathrm{s}} \leq R \tilde{x} \leq u_{\mathrm{s}}\right\}
$$

Assumption 1: The nonlinearity $\psi(\tilde{u})=q u a(\tilde{u})-u$ satisfies conditions $\square$

$$
\begin{gathered}
\psi(\tilde{u})^{T} T_{2} \tilde{u}+\Delta \mathbf{1}_{m}^{T} T_{2}|\tilde{u}| \geq 0, \tilde{u} \in \mathbf{R}^{m} \\
\psi(\tilde{u})^{T} T_{3}(\psi(\tilde{u})+\tilde{u}) \leq 0, \tilde{u} \in \mathbf{R}^{m} \\
(\psi(\tilde{u})+\tilde{u})^{T} T_{4}(\psi(\tilde{u})-\tilde{u}) \leq 0, \tilde{u} \in \mathbf{R}^{m}
\end{gathered}
$$

for any diagonal positive matrices $T_{2}, T_{3}, T_{4} \in \mathbf{R}^{m \times m}$.

For system $\dot{\tilde{x}}(t)=[A(\theta)+B(\theta) K] \tilde{x}(t)+B(\theta)[\phi(K \tilde{x}(t))+\psi(K \tilde{x}(t))]$, with the satisfaction of conditions (19) and (21), the following proposition for the design of a gain $K$ can be stated by using Assumption 1, Assumption 2 and Lyapunov stability theory.

Proposition 1: If there exist two symmetric positive definite matrices $W, Q \in \mathbf{R}^{n \times n}$, four diagonal positive matrices $S_{1}, S_{2}, S_{3}, S_{4} \in \mathbf{R}^{m \times m}$, two matrices $Y, Z \in \mathbf{R}^{m \times n}$, and two positive scalars $\tau_{1}, \tau_{2}$, satisfying

$$
\begin{aligned}
& {\left[\begin{array}{ccccc}
H e\left(A_{i} W+B_{i} Y\right)-\tau_{1} W+\tau_{2} Q & * & * & * & * \\
S_{1} B_{i}^{T}-Y-Z & -2 S_{1} & * & * & * \\
S_{3} B_{i}^{T}-Y+S_{3} S_{2}^{-1} Y & -S_{3} & -2 S_{3}-S_{3} S_{4}^{-1} S_{3} & * & * \\
\Delta Y & \mathbf{0} & \mathbf{0} & \left(\tau_{1}-\tau_{2}\right) S_{2}^{2} / m & * \\
Y & \mathbf{0} & \mathbf{0} & \mathbf{0} & -S_{4}
\end{array}\right]<\mathbf{0}, i=1,2, \ldots, N} \\
& {\left[\begin{array}{cc}
W & Z^{T} \\
Z & u_{s}^{2}
\end{array}\right] \geq \mathbf{0}}
\end{aligned}
$$




$$
\begin{gathered}
Q-W \geq \mathbf{0} \\
{\left[\begin{array}{ccc}
H e\left(A_{i} W+B_{i} Y\right) & * & * \\
B_{i}^{T} & -\gamma_{1} I & * \\
-H W & 0 & 1-\gamma I
\end{array}\right]<\mathbf{0}, i=1,2, \ldots, N} \\
{\left[\begin{array}{cccc}
H e\left(A_{i} W+B_{i} Y\right) & * & * & * \\
0 & H e\left(A_{j} W+B_{j} Y\right) & * & * \\
B_{i}^{T} & B_{j}^{T} & -\gamma_{2} I & * \\
-H_{i} W & H_{j} W & 0 & -\gamma_{2} I
\end{array}\right]<\mathbf{0}, i=1,2, \ldots, N-1, j=i+1}
\end{gathered}
$$

then $K=Y W^{l}$ is a stabilizing gain for the time-varying system with smooth switching, and the sets $S_{0}$ and $S_{\infty}$ are defined by

$$
\begin{gathered}
S_{0}=\left\{\tilde{x} \in \mathbf{R}^{n} ; \tilde{x}^{T} P_{1} \tilde{x} \leq 1\right\}, P_{1}=W^{-1} \\
S_{\infty}=\left\{\tilde{x} \in \mathbf{R}^{n} ; \tilde{x}^{T} P_{2} \tilde{x} \leq 1\right\}, P_{2}=W^{-1} Q W^{-1}
\end{gathered}
$$

where the domain of attraction is $S_{0} \backslash S_{\infty}$ and the domain of convergence is $S_{\infty} \cdot \gamma_{1}$ and $\gamma_{2}$ are the performance indexes, which should be minimum, so as to achieve smooth control.

Proof: For the closed-loop system $\dot{\tilde{x}}(t)=[A(\theta)+B(\theta) K] \tilde{x}(t)+B(\theta)[\phi(K \tilde{x}(t))+\psi(K \tilde{x}(t))]$, consider the quadratic Lyapunov function $V(\tilde{x})=\tilde{x}^{T} P_{1} \tilde{x}$, by Ref. [17], the inequality (23) and the following inequality (27) are satisfied.

$$
\left[\begin{array}{ccccc}
H e[A(\theta) W+B(\theta) Y]-\tau_{1} W+\tau_{2} Q & * & * & * & * \\
S_{1} B(\theta)^{T}-Y-Z & -2 S_{1} & * & * & * \\
S_{3} B(\theta)^{T}-Y+S_{3} S_{2}^{-1} Y & -S_{3} & -2 S_{3}-S_{3} S_{4}^{-1} S_{3} & * & * \\
\Delta Y & \mathbf{0} & \mathbf{0} & \left(\tau_{1}-\tau_{2}\right) S_{2}^{2} / m & * \\
Y & \mathbf{0} & \mathbf{0} & \mathbf{0} & -S_{4}
\end{array}\right]<\mathbf{0}
$$

According to Ref. [17], the stabilizing gain $\mathrm{K}=\mathrm{YW}^{-1}$ can be found and for any $\tilde{x}$, that satisfy $\tilde{X}^{T} P_{1} \tilde{X} \leq 1$, and $\tilde{x}^{T} P_{2} \tilde{X} \geq 1$, one can obtained that $\dot{V}(\tilde{x}) \leq-\zeta(V(\tilde{x}))$, where $\zeta($.$) is a class k$ function.

By using $A(\theta)=\sum_{i=1}^{N} \theta_{i} A_{i}, \quad B(\theta)=\sum_{i=1}^{N} \theta_{i} B_{i}$, and Eq.(27), we have[

$$
\left[\begin{array}{ccccc}
H e\left[\sum_{i=1}^{N} \theta_{i} A_{i} W+\sum_{i=1}^{N} \theta_{i} B_{i} Y\right]-\tau_{1} W+\tau_{2} Q & * & * & * & * \\
S_{1} \sum_{i=1}^{N} \theta_{i} B_{i}^{T}-Y-Z & -2 S_{1} & * & * & * \\
S_{3} \sum_{i=1}^{N} \theta_{i} B_{i}^{T}-Y+S_{3} S_{2}^{-1} Y & -S_{3} & -2 S_{3}-S_{3} S_{4}^{-1} S_{3} & * & * \\
\Delta Y & \mathbf{0} & \mathbf{0} & \left(\tau_{1}-\tau_{2}\right) S_{2}^{2} / m & * \\
Y & \mathbf{0} & \mathbf{0} & \mathbf{0} & -S_{4}
\end{array}\right]<\mathbf{0}
$$

Since $\sum_{i=1}^{N} \theta_{i}=1$, then

$$
\sum_{i=1}^{N} \theta_{i}\left[\begin{array}{ccccc}
H e\left[A_{i} W+B_{i} Y\right]-\tau_{1} W+\tau_{2} Q & * & * & * & * \\
S_{1} B_{i}^{T}-Y-Z & -2 S_{1} & * & * & * \\
S_{3} B_{i}^{T}-Y+S_{3} S_{2}^{-1} Y & -S_{3} & -2 S_{3}-S_{3} S_{4}^{-1} S_{3} & * & * \\
\Delta Y & \mathbf{0} & \mathbf{0} & \left(\tau_{1}-\tau_{2}\right) S_{2}^{2} / m & * \\
Y & \mathbf{0} & \mathbf{0} & \mathbf{0} & -S_{4}
\end{array}\right]<\mathbf{0}
$$


Therefore, the satisfaction of inequality (23) implies the above inequality.

Since the domain of convergence should be included in the domain of attraction, that is $\tilde{x}^{T} P_{1} \tilde{x} \leq \tilde{X}^{T} P_{2} \tilde{X} \leq 1$, then $P_{2}-P_{1}=W^{-1} Q W^{-1}-1 \geq 1$, one can get that $\mathrm{Q}-\mathrm{W} \geq 0$. So the inequality (24) is satisfied.

By using Bounded Real Lemma [19], the condition $\left\|G_{\theta}(s)\right\|_{\infty}<\gamma_{1}$ described in (19) can be transformed as follows

$$
\left[\begin{array}{ccc}
H e[A(\theta) W+B(\theta) Y] & * & * \\
B(\theta)^{T} & -\gamma_{1} I & * \\
-H(\theta) W & 0 & -\gamma_{1} I
\end{array}\right]<\mathbf{0}
$$

Then by $\operatorname{using} A(\theta)=\sum_{i=1}^{N} \theta_{i} A_{i}, \quad B(\theta)=\sum_{i=1}^{N} \theta_{i} B_{i}$ and $H(\theta)=\sum_{i=1}^{N} \theta_{i} H_{i}$, inequality (29) can be rewritten as

$$
\left[\begin{array}{ccc}
H e\left[\sum_{i=1}^{N} \theta_{i} A_{i} W+\sum_{i=1}^{N} \theta_{i} B_{i} Y\right] & * & * \\
\sum_{i=1}^{N} \theta_{i} B_{i}^{T} & -\gamma_{1} I & * \\
-\sum_{i=1}^{N} \theta_{i} H_{i} W & 0 & -\gamma_{1} I
\end{array}\right]<\mathbf{0}
$$

which is equivalent to

$$
\sum_{i=1}^{N} \theta_{i}\left[\begin{array}{ccc}
H e\left(A_{i} W+B_{i} Y\right) & * & * \\
B_{i}^{T} & -\gamma_{1} I & * \\
-H W & 0 & -\gamma_{1} I
\end{array}\right]<\mathbf{0}, i=1,2, \ldots, N
$$

The satisfaction of inequality (25) implies the above inequality, so the condition $\left\|G_{\theta}(s)\right\|_{\infty}<\gamma_{1}$ can be satisfied.

By the Bounded eal Lemma [19], the condition $\left\|G_{\beta_{m}}(s)-G_{\beta n}(s)\right\|_{\infty}<\gamma_{2}$ described in (21) can be transformed as follows:

$$
\left[\begin{array}{cccc}
H e\left[A\left(\beta_{m}\right) W+B\left(\beta_{m}\right) Y\right] & * & * & * \\
0 & H e\left[A\left(\beta_{n}\right) W+B\left(\beta_{n}\right) Y\right] & * & * \\
B\left(\beta_{m}\right)^{T} & B\left(\beta_{n}\right)^{T} & -\gamma_{2} I & * \\
-H\left(\beta_{m}\right) W & H\left(\beta_{n}\right) W & 0 & -\gamma_{2} I
\end{array}\right]<\mathbf{0}
$$

The above inequality can be rewritten as:

$$
\left[\begin{array}{cccc}
H e\left[A\left(\beta_{m}\right) W+B\left(\beta_{m}\right) Y\right] & * & * & * \\
0 & 0 & * & * \\
B\left(\beta_{m}\right)^{T} & 0 & -\gamma_{2} I & * \\
-H\left(\beta_{m}\right) W & 0 & 0 & -\gamma_{2} I
\end{array}\right]+\left[\begin{array}{cccc}
0 & * & * & * \\
0 & H e\left[A\left(\beta_{n}\right) W+B\left(\beta_{n}\right) Y\right] & * & * \\
0 & B\left(\beta_{n}\right)^{T} & 0 & * \\
0 & H\left(\beta_{n}\right) W & 0 & 0
\end{array}\right]<\mathbf{0}
$$

which is equivalent to:

$$
\sum_{i=1}^{N} \beta_{m i}\left[\begin{array}{cccc}
H e\left[A_{i} W+B_{i} Y\right] & * & * & * \\
0 & 0 & * & * \\
B_{i}^{T} & 0 & -\gamma_{2} I & * \\
-H_{i} W & 0 & 0 & -\gamma_{2} I
\end{array}\right]+\sum_{i=1}^{N} \beta_{n i}\left[\begin{array}{cccc}
0 & * & * & * \\
0 & H e\left[A_{i} W+B_{i} Y\right] & * & * \\
0 & B_{i}^{T} & 0 & * \\
0 & H_{i} W & 0 & 0
\end{array}\right]<\mathbf{0}
$$

Since $\sum_{i=1}^{N} \beta_{m i}=1$ and $\sum_{i=1}^{N} \beta_{n i}=1$ by inequality (34), one can get that 


$$
\sum_{i=1}^{N} \beta_{n i} \sum_{i=1}^{N} \beta_{m i}\left[\begin{array}{cccc}
H e\left[A_{i} W+B_{i} Y\right] & * & * & * \\
0 & 0 & * & * \\
B_{i}^{T} & 0 & -\gamma_{2} I & * \\
-H_{i} W & 0 & 0 & -\gamma_{2} I
\end{array}\right]+\sum_{i=1}^{N} \beta_{n i} \sum_{i=1}^{N} \beta_{m i}\left[\begin{array}{cccc}
0 & * & * & * \\
0 & H e\left[A_{i} W+B_{i} Y\right] & * & * \\
0 & B_{i}^{T} & -\gamma_{2} I & * \\
0 & H_{i} W & 0 & -\gamma_{2} I
\end{array}\right]<\mathbf{0}
$$

Then,

$$
\sum_{i=1}^{N} \beta_{n i} \sum_{i=1}^{N} \beta_{m i}\left\{\left[\begin{array}{cccc}
H e\left[A_{i} W+B_{i} Y\right] & * & * & * \\
0 & 0 & * & * \\
B_{i}^{T} & 0 & -\gamma_{2} I & * \\
-H_{i} W & 0 & 0 & -\gamma_{2} I
\end{array}\right]+\left[\begin{array}{cccc}
0 & * & * & * \\
0 & H e\left[A_{i} W+B_{i} Y\right] & * & * \\
0 & B_{i}^{T} & -\gamma_{2} I & * \\
0 & H_{i} W & 0 & -\gamma_{2} I
\end{array}\right]\right\}<\mathbf{0}
$$

The satisfaction of inequality (26) in Proposition 1 implies the above inequality, so the condition $\left\|G_{\beta_{m}}(s)-G_{\beta n}(s)\right\|_{\infty}<\gamma_{2}$ can be satisfied. Thus, the proof is complete.

Remark 1: It is important to note that Eq. (22) is nonlinear in the decision variables, which prevents from solving directly a convex optimization problem. Therefore, the following additional constraints are considered in the decision variables.

$$
S_{2}=S_{3}, \quad S_{4}=\mathbf{1} / \tau_{4}, \quad S_{3} \geq \tau_{3} \mathbf{1}
$$

Then, inequality (22) can be transformed to be inequality (37)

$$
\left[\begin{array}{ccccc}
H e\left(A_{i} W+B_{i} Y\right)-\tau_{1} W+\tau_{2} Q & * & * & * & * \\
S_{1} B_{i}^{T}-Y-Z & -2 S_{1} & * & * & * \\
S_{3} B_{i}^{T} & -S_{3} & -\left(2+\tau_{3} \tau_{4}\right) S_{3} & * & * \\
\Delta Y & \mathbf{0} & \mathbf{0} & \left(\tau_{1}-\tau_{2}\right) \tau_{3} S_{3} / m & * \\
\tau_{4} Y & \mathbf{0} & \mathbf{0} & \mathbf{0} & -\tau_{4} \mathbf{1}
\end{array}\right]<\mathbf{0}, i=1,2, \ldots, N
$$

Remark 2: In order to obtain an outer set $S_{0}$ as large as possible and an inner set $S_{\infty}$ as small as possible, two additional inequalities as Eq.(38) and Eq.(39) are introduced

$$
\begin{gathered}
{\left[\begin{array}{cc}
\Omega & v_{r}^{T} \\
v_{r} & W
\end{array}\right] \geq \mathbf{0}} \\
{\left[\begin{array}{cc}
Q & -W \\
-W & X
\end{array}\right] \geq \mathbf{0}}
\end{gathered}
$$

where $\mathrm{v}_{\mathrm{r}}$ is a vector whose size and direction are used to orient the ellipsoid $S_{0}, \Omega$ and $H$ are sets as optimization parameters to make a convenient compromise between a large $S_{0}$ and a small $S_{\infty}$.

Considering $\tau_{1}, \tau_{2}, \tau_{3}$ and $\tau_{4}$ as tuning parameters, $\Omega$ and $H$ as optimization parameters, the optimization problem with input saturation and input quantization is stated as follows:

$$
\min \left\{\operatorname{trace}(H)+\Omega+\gamma_{1}+\gamma_{2}\right\}
$$

subject to Eqs. (23-26), Eqs. (37-39) and $S_{3} \geq \tau_{3} 1$.

\section{SIMULATIONS}

In this paper, the longitudinal dynamics model of hypersonic vehicle will be considered as:

$$
\left\{\begin{array}{l}
\dot{x}=A x+B u \\
z=H x
\end{array}\right.
$$


with system state $x=\left[\begin{array}{ll}\alpha & q\end{array}\right]^{T}$, where $\alpha$ is angle-of-attack and $q$ is pitch rate. $A$ is system matrix. $B$ is control matrix, representing the force and moment of RCS. Control input $\mathrm{u}$ is the RCS firing command, standing for the number and direction of thrusters fired. It is assumed that the maximum force produced by RCS is $8 \mathrm{~F}$, and the bound of quantization error is $F$, that is $\mathrm{u}_{\mathrm{s}}=8$ and $\Delta=1$.

The system performance output is angle-of-attack, that is:

$$
H=\left[\begin{array}{ll}
1 & 0
\end{array}\right]
$$

The system matrices and control matrices of longitudinal linear dynamics model of hypersonic vehicle at five different trim points are stated as follows:

Model 1:

$$
A_{1}=\left[\begin{array}{cc}
-0.0014 & 1 \\
0.1122 & -0.0024
\end{array}\right], \quad B_{1}=\left[\begin{array}{l}
0.000034 \\
0.019863
\end{array}\right]
$$

Model 2:

$$
A_{2}=\left[\begin{array}{rc}
-0.0021 & 1 \\
0.1336 & -0.0032
\end{array}\right], B_{2}=\left[\begin{array}{l}
0.000036 \\
0.019863
\end{array}\right]
$$

Model 3:

$$
A_{3}=\left[\begin{array}{rc}
-0.0022 & 1 \\
0.1689 & -0.0040
\end{array}\right], B_{3}=\left[\begin{array}{l}
0.000039 \\
0.019863
\end{array}\right]
$$

Model 4:

$$
A_{4}=\left[\begin{array}{cc}
-0.0027 & 1 \\
0.1927 & -0.0048
\end{array}\right], \quad B_{4}=\left[\begin{array}{l}
0.000043 \\
0.019863
\end{array}\right]
$$

Model 5:

$$
A_{5}=\left[\begin{array}{rc}
-0.0042 & 1 \\
0.2230 & -0.0066
\end{array}\right], \quad B_{5}=\left[\begin{array}{l}
0.000053 \\
0.019863
\end{array}\right]
$$

Selecting turning parameters of LMIs as $\tau_{1}=10^{-7}, \tau_{2}=0.185, \tau_{3}=50, \tau_{4}=10^{-3}$, and solving the convex optimization problem of Eq. (40), we find the state feedback gain as:

$$
K=\left[\begin{array}{lll}
-708.7414 & -387.1565 & 438.3598
\end{array}\right]
$$

If the condition of smooth switching is not considered, that is the inequality (26) will be ignored, selecting the turning parameters be the same as above, one can obtain that the state feedback gain as:

$$
K=\left[\begin{array}{lll}
-471.2527 & -260.9527 & 288.3237
\end{array}\right]
$$

Supposing the tracking command of angle-of-attack is 10 degree, the initial angle-of-attack is 0 degree and the initial pitch rate is 0 degree/s. The initial system model is model 1 , at the simulation time of 20 s, the system model switches from model 1 to model 5. The simulation results with continuous input and discrete input are given as follows, respectively. 


\subsection{Simulation Results With Continuous Input}

Figs. (3-5) present the curves of angle-of-attack, pitch rate and the assumed continuous RCS control command, in which, the solid line represents the results using controller (41) with the condition of smooth switching and the dashed line is the results using controller (42) without the condition of smooth switching.

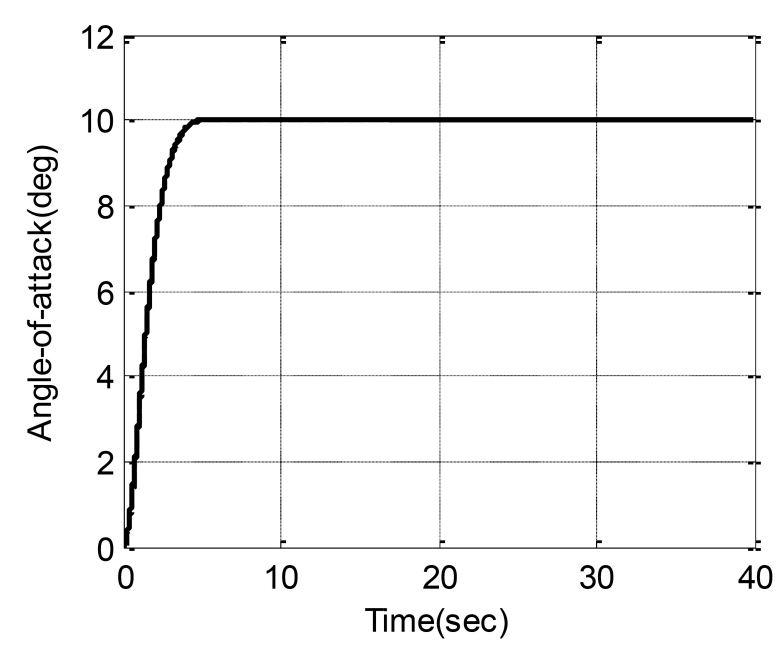

(a) Original figure

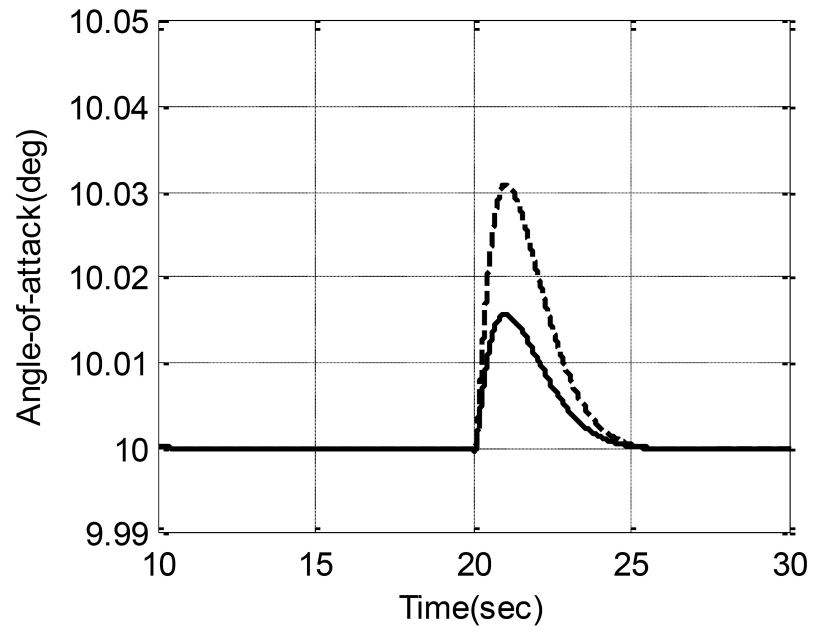

(b) Enlarged figure

Fig. (3). Angle-of-attack (original figure and enlarged figure in 10s-30s).

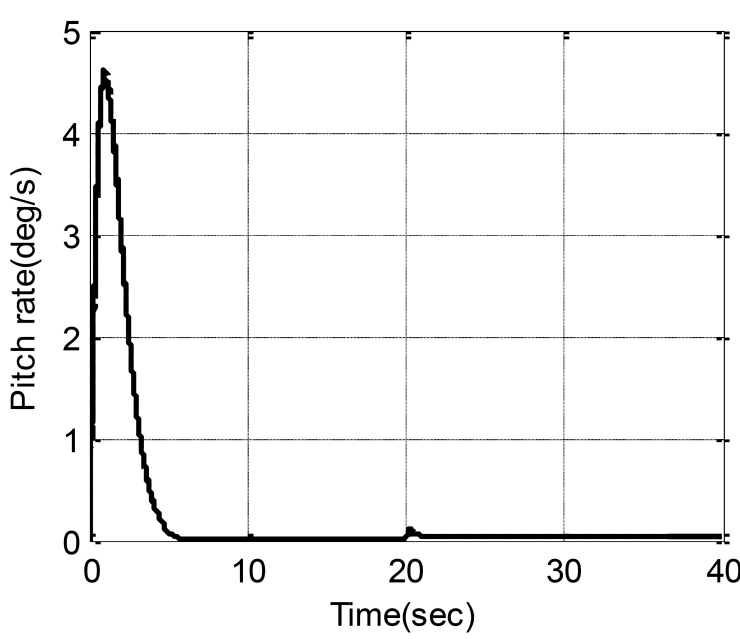

(a) Original figure

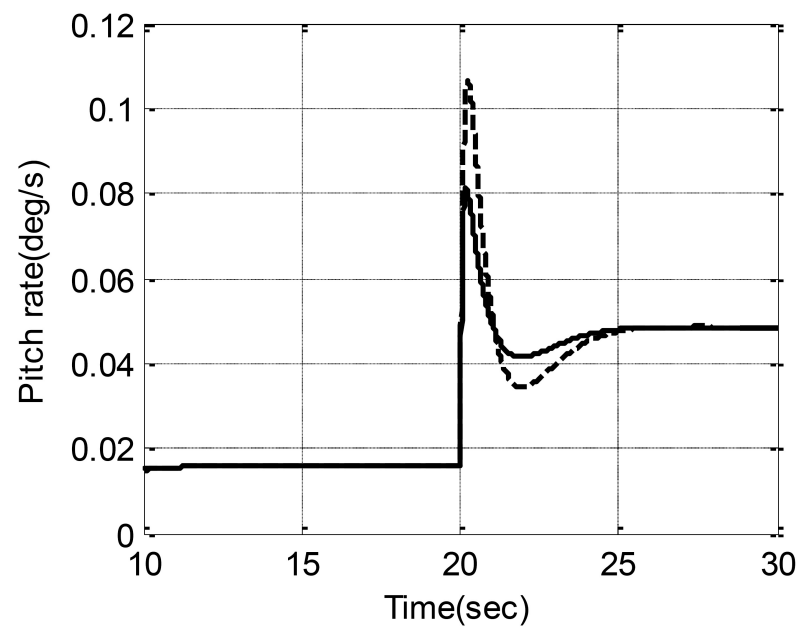

(b) Enlarged figure

Fig. (4). Pitch rate (original figure and enlarged figure in 10s-30s). 


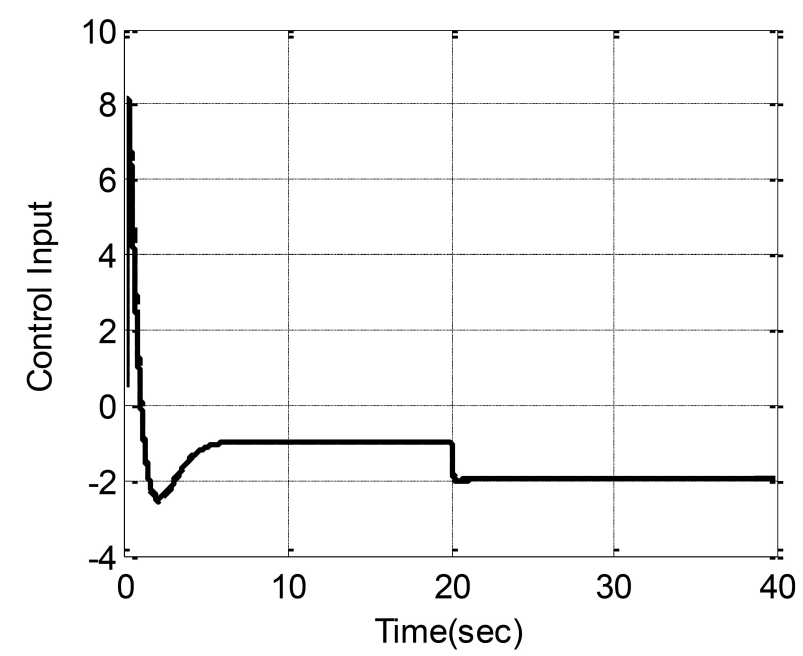

(a) Original figure

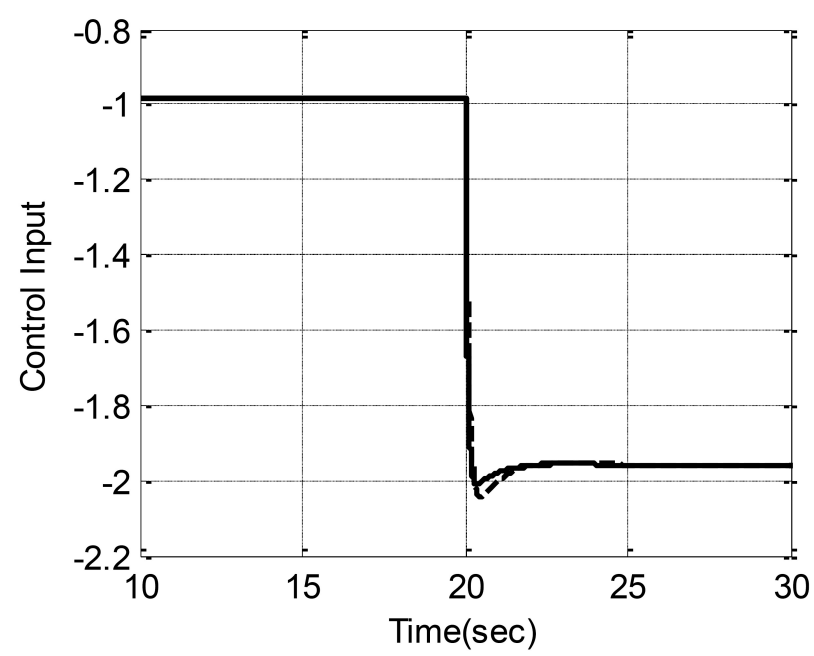

(b) Enlarged figure

Fig. (5). RCS control command (original figure and enlarged figure in 10s-30s).

\subsection{Simulation Results with Discrete Input}

The curves of angle-of-attack, pitch rate and the discrete RCS control command are presented in Figs. (6-9), in which, the solid lines in Figs. (6-8) represent the results using controller (41) with the condition of smooth switching and the dashed line in Figs. $(\mathbf{6}, 7,9)$ is the results using controller $(42)$ without the condition of smooth switching.

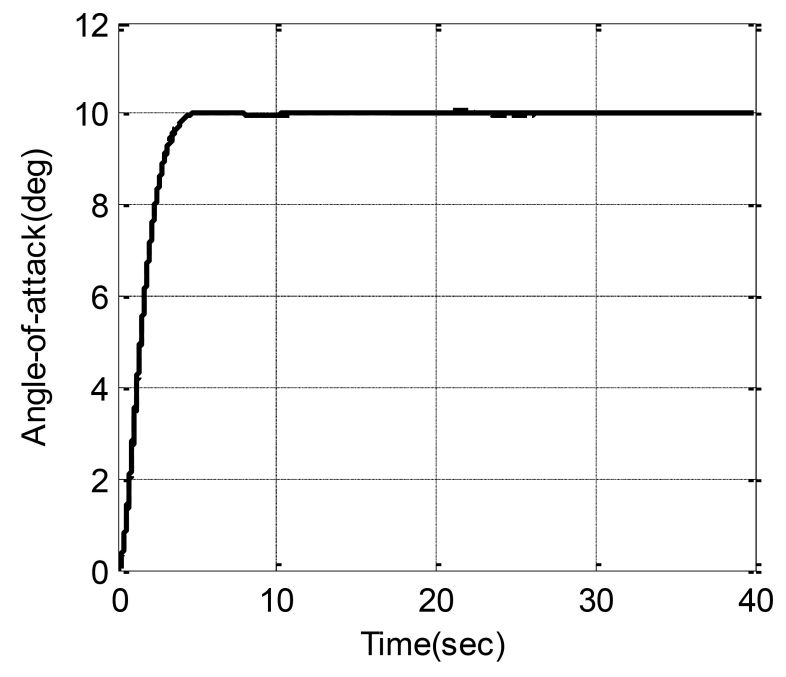

(a) Original figure

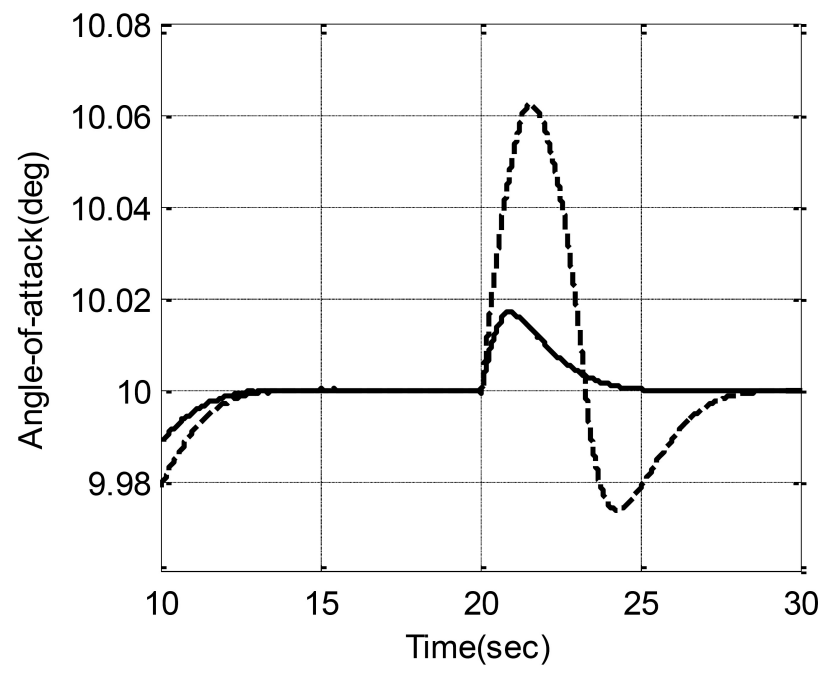

(b) Enlarged figure

Fig. (6). RCS control command (original figure and enlarged figure in 10s-30s).

It can be seen from Figs. (3-9) that, the tracking performance satisfies the practical specifications, and the state feedback controller obtained by this proposed method can track a given reference signal well in the considered flight envelop. And also, the switching vibration occurring in variation of the parameter is suppressed effectively by our proposed method. 


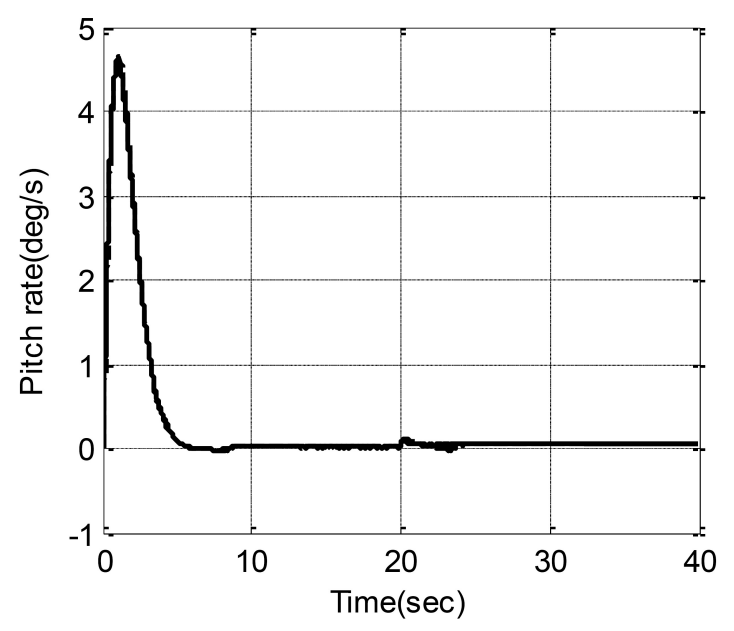

(a) Original figure

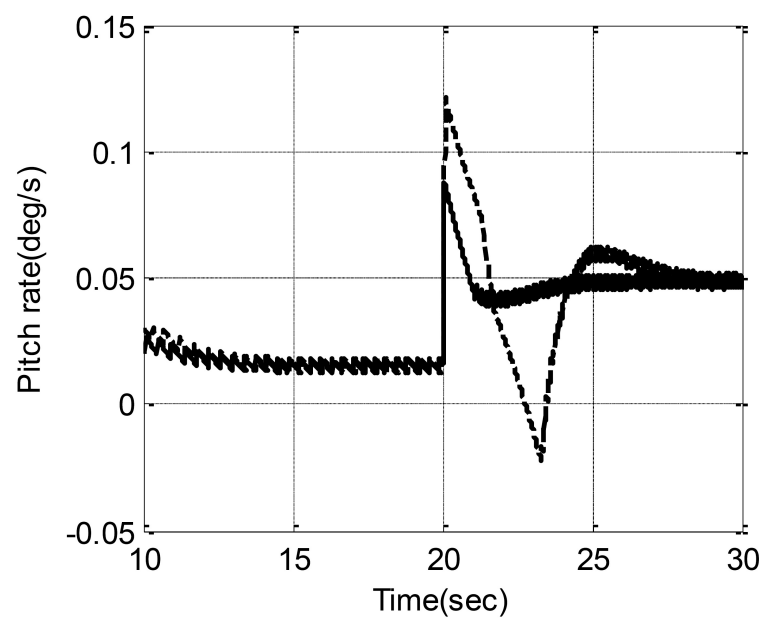

(b) Enlarged figure

Fig. (7). Pitch rate (original figure and enlarged figure in 10s-30s).
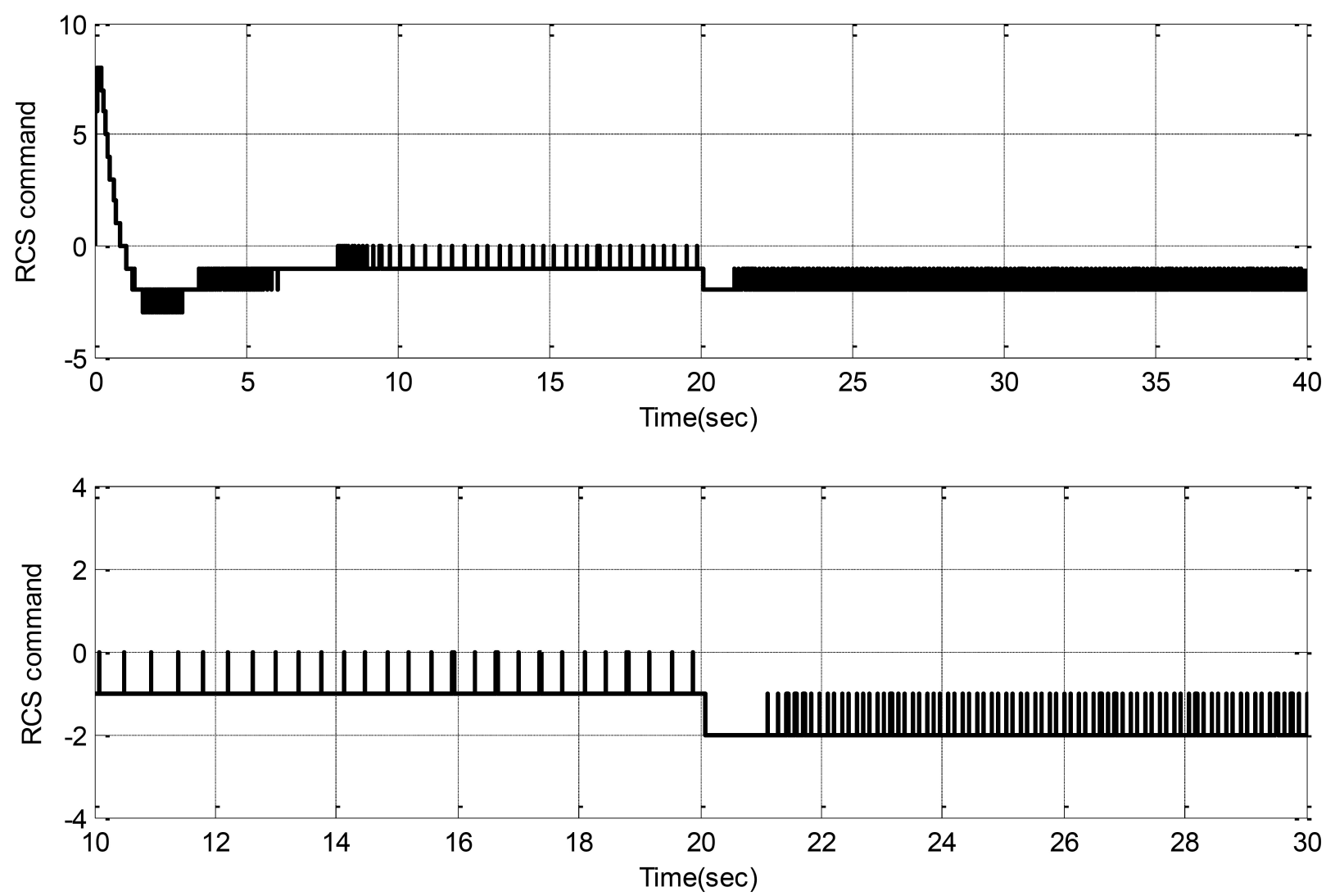

Fig. (8). RCS control command with the condition of smooth switching (original figure and enlarged figure). 

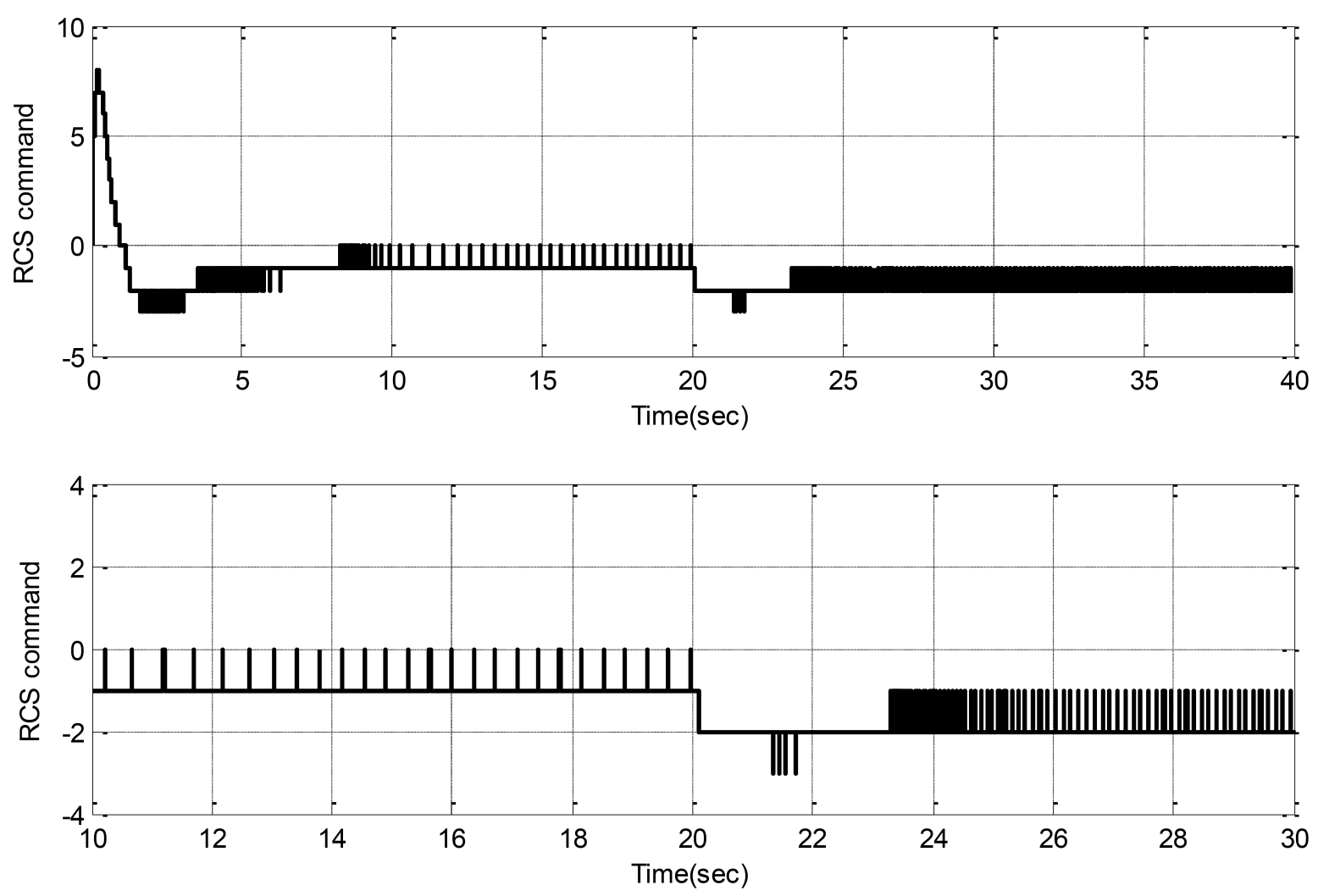

Fig. (9). RCS control command without the condition of smooth switching (original figure and enlarged figure).

\section{CONCLUSION}

This paper has investigated the problem of smooth switching state feedback controller design for linear time-varying systems with input quantization and input Saturation. By constructing a deviation system, a command-tracking problem can be converted to a regulator problem. By using robust control technique, the conditions for smooth switching steady tracking control are presented. Then, with the help of Lyapunov stability theory, the state feedback gain of the deviation system with input saturation and input quantization is designed by solving Linear Matrix Inequalities (LMIs) based convex optimization problem with the conditions for smooth switching tracking control being considered. Simulations results using the longitudinal dynamics models of hypersonic vehicle driven by Reaction Control System (RCS) reveal that, the system designed using the smooth switching tracking method proposed in this paper has better transient behavior when system parameter variation occurs.

\section{CONFLICT OF INTEREST}

The authors confirm that this article content have no conflict of interest.

\section{ACKNOWLEDGEMENTS}

Declare none.

\section{REFERENCES}

[1] J.F. Shamma, and J.R. Cloutier, "A linear parameter varying approach to gain scheduled missile autopilot design", Proceeding of American Control Conference, 1992 Chicago, IL, USA

[2] P. Apkarian, P. Gahinet, and G. Becker, "Self-scheduled Ho control of linear parameter-varying systems: A design example", Automatica., vol. 31, pp. 1251-1261, 1995.

[http://dx.doi.org/10.1016/0005-1098(95)00038-X]

[3] J.F. Magni, "Multi-model eigenstructure assignment in flight control design", Aerosp. Sci. Technol., vol. 3, no. 3, pp. 141-151, 1999. [http://dx.doi.org/10.1016/S1270-9638(99)80038-8]

[4] G.K. Thampi, J.C. Prncipe, M.A. Motter, J.H. Cho, and J. Lan, "Multiple model based flight control design", In: Midwest Symposium on 
Circuits and Systems. 2002, pp. 133-136.

[5] F. Liao, J.L. Wang, and G.H. Yang, "Reliable robust flight tracking control: an LMI approach", IEEE Trans. Contr. Syst. Technol., vol. 10, no. 1 , pp. 76-89, 2002.

[http://dx.doi.org/10.1109/87.974340]

[6] S.L. Dai, G.M. Dimirovski, and J. Zhao, "A descriptor system approach to robust Hœ control and its application to flight control", In: Proceedings of American Control Conference. 2006.Minneapolis, MN, USA

[7] G. H. Cai, J. M. Song, and X. X. Chen, "Command tracking control system design and evaluation for hypersonic reentry vehicles driven by a reaction control system", J. Aero. Eng., 2014. 10.1061/(ASCE)AS.1943-5525.0000448, 04014115, Published online

[8] Anon, "Guidance System Operations Plan for Manned CM Earth Orbital and Lunar Missions Using Program Colossus 3, Sec. 3, Digital Autopilots (Rev. 14)", NASA Tech. Rept. MIT IL R-577, . Contract NAS 9-4065

[9] P.D. Hattis, "Qualitative differences between shuttle on-orbit and transition control", J. Guid. Control Dyn., vol. 7, no. 1, pp. 4-8, 1984. [http://dx.doi.org/10.2514/3.56361]

[10] N. Alexander, P.D. Penchuk, and E.T. Kubiak, "Frequency domain stability analysis of a phase plane control system", J. Guid. Control Dyn., vol. 8 , no. 1 , pp. 50-55, 1985 . [http://dx.doi.org/10.2514/3.19934]

[11] P.C. Calhoun, and E.M. Queen, "Entry vehicle control system design for the mars science laboratory", J. Spacecr. Rockets, vol. 43, no. 2, pp. 324-329, 2006.

[http://dx.doi.org/10.2514/1.19650]

[12] J.A. Paradiso, "Adaptable method of managing jets and aerosurfaces for aerospace vehicle control", J. Guid. Control Dyn., vol. 14, no. 1, pp. 44-50, 1991.

[http://dx.doi.org/10.2514/3.20603]

[13] S.W. Thurman, and H. Flashner, "Robust digital autopilot design for spacecraft equipped with pulse operated thrusters", J. Guid. Control Dyn., vol. 19, no. 5, pp. 1047-1055, 1996. [http://dx.doi.org/10.2514/3.21744]

[14] G.H. Cai, J.M. Song, and X.X. Chen, "Control system design for hypersonic reentry vehicle driven by aerosurfaces and reaction control system", Proc. Inst. Mech. Eng. Part G J. Aerosp. Eng., vol. 229, no. 9, pp. 1575-1587, 2014. [http://dx.doi.org/10.1177/0954410014555894]

[15] M. Hanifzadegan, and R. Nagamune, "Smooth switching LPV controller design for LPV systems", Automatica, no. 50, pp. 1481-1488, 2014. [http://dx.doi.org/10.1016/j.automatica.2014.03.014]

[16] G. Battistelli, E. Mosca, and P. Tesi, "Adaptive memory in multi-model switching control of uncertain plants", Automatica, no. 50, pp. 874-882, 2014.

[http://dx.doi.org/10.1016/j.automatica.2013.12.002]

[17] S. Tarbouriech, and F. Gouaisbaut, "Control design for quantized linear systems with saturations", IEEE Trans. Automat. Contr., vol. 57, no. 7, pp. 1883-1889, 2012. [http://dx.doi.org/10.1109/TAC.2011.2179845]

[18] L. Song, and J.J. Yang, "Smooth switching output tracking control for lpv systems", Asian J. Control, vol. 14, no. 6, pp. 1710-1716, 2012. [http://dx.doi.org/10.1002/asjc.510]

[19] G. Michael, and J.N. David, Linear Robust Control. Pearson Education, Inc.: London, 1995.

(C) Hu et al.; Licensee Bentham Open.

This is an open access article licensed under the terms of the Creative Commons Attribution-Non-Commercial 4.0 International Public License (CC BY-NC 4.0) (https://creativecommons.org/licenses/by-nc/4.0/legalcode), which permits unrestricted, non-commercial use, distribution and reproduction in any medium, provided the work is properly cited. 